\title{
Fear of relapse, social support, and psychological well-being (depression, anxiety, and stress level) of patients with multiple sclerosis (MS) during the COVID-19 pandemic stage
}

\author{
Vahid Shaygannejad $^{1,2} \cdot$ Omid Mirmosayyeb $b^{1,2,3} \cdot$ Nasim Nehzat $^{2} \cdot$ Mahsa Ghajarzadeh $^{3,4}$ (D)
}

Received: 20 January 2021 / Accepted: 10 April 2021 / Published online: 15 April 2021

(C) Fondazione Società Italiana di Neurologia 2021

\begin{abstract}
Background Psychological well-being assessment during the COVID-19 pandemic is essential for patients with multiple sclerosis (MS). The goal of this study is to evaluate fear of relapse, social support, and psychological well-being (depression, anxiety, and stress level) of Iranian patients with MS during the COVID-19 pandemic stage.

Methods One hundred and sixty-five patients were enrolled. We asked all cases to fill valid and reliable Persian version of depression, anxiety, and stress scale (DASS-21), perceived social support, and fear of relapse scale questionnaires.

Results One hundred and sixty-five patients were enrolled. Female to male ratio was $(F / M)=4.6$. Mean age and mean duration of disease were $35.3 \pm 8.6$ and 7.1 \pm 5 years, respectively. Mean scores of social support, DASS, and FoR questionnaires were 63.1 $\pm 16.8,16.4 \pm 13.4$, and $51.4 \pm 17.3$, respectively. There was a significant negative correlation between social support and FoR scores and also significant positive correlations between components of DASS and FoR. Linear regression analysis by considering FoR as dependent variable and age, sex, marital status, duration of the disease, and EDSS as dependent variables showed that sex was an independent predictor of FoR score.

Conclusion Psychological well-being as well as fear of relapse should be considered in patients with MS during the COVID-19 pandemic stage.
\end{abstract}

Keywords Relapse $\cdot$ Multiple sclerosis $\cdot$ Anxiety $\cdot$ Stress $\cdot$ COVID-19

\section{Introduction}

Multiple sclerosis is an autoimmune disease of the central nervous system which has effects on all aspect of life [1,2]. The most common form of the disease is relapsing-remitting (RR) $(85 \%)$ which is characterized by relapses (newly developed neurological symptoms or worsening of previous ones)

Mahsa Ghajarzadeh

m.ghajarzadeh@gmail.com

1 Department of Neurology, School of Medicine, Isfahan University of Medical Sciences, Isfahan, Iran

2 Isfahan Neurosciences Research Center, Isfahan University of Medical Sciences, Isfahan, Iran

3 Universal Council of Epidemiology (UCE), Universal Scientific Education and Research Network (USERN), Tehran University of Medical Sciences, Tehran, Iran

4 Multiple Sclerosis Research Center Neuroscience Institute, Tehran University of Medical Sciences, Tehran, Iran and non-relapse phases [3-5]. Ozakbas et al. reported that patient in the relapse phase of the disease has impaired quality of life than cases without relapses [6].

Anxiety is common in chronic illnesses such as MS [7] which is related with disability level [8]. The role of stress in pathogenesis of MS is clear and stress level is related with worsening of the neurological manifestations [9]. Fear is the core part of anxious thoughts and MS patients experience fear especially in relapse phases, based on the unpredictable nature of the relapses $[5,10]$. On the other hand, fear of progression of disabilities in MS is protruding and the patients need psychological support.

Recently, Khatibi et al. developed [10] a questionnaire that measures fear of relapses and disease activity in MS patients which seems to be useful for psychological evaluation of the patients and determines the possible leading factors. During the COVID-19 pandemic stage, patients with MS suffer more from anxiety due to worries of disease management and access to health care services in this period and more depression than healthy subjects [11]. On 
the other hand, physicians face challenges with monitoring of their patients, their disability status, and medication efficacy [12].

We designed this study during the COVID-19 pandemic stage to evaluate fear of relapse, social support, and psychological well-being (depression, anxiety, and stress level) of Iranian patients with MS in this period.

\section{Methods}

This cross-sectional study was conducted in Kashani Hospital of Isfahan University of Medical Sciences between June and September 2020.

Inclusion criteria were age more than 18 and RR form of the disease.

Exclusion criteria were other forms of MS and no relapses during the last month.

All patients were asked to fill informed consent forms and the study was approved by the local ethics committee.

We asked all cases to fill valid and reliable Persian version of the depression, anxiety, and stress scale (DASS-21), perceived social support, and fear of relapse scale [10, 13, 14].

DASS-21 consists of 21 questions evaluating depression, anxiety, and stress. Each item is evaluated by 7 questions. Questions are rated on a 4-point Likert scale $(0=$ did not apply to me at all; $3=$ applied to me very much or most of the time). Sum of the scores of each item shows the final score. The higher the total scores, the higher levels of depression, anxiety, and stress symptoms [13].

Perceived social support includes 12 questions which assesses different categories of support. Each item is graded from 1 (very strongly disagree) to 7 (very strongly). The sum of the scores shows the final score [14].

Fear of relapse scale includes 26 questions each scored on a five-Likert scale ranging from $1=$ never, $2=$ rarely, $3=$ sometimes, $4=$ often, and $5=$ always. Three components were suggested by the authors: feeling related to another relapse, physiological effects of relapse, and fear associated with side effects of a relapse. The total score was the sum of the three components [10].

All data were analyzed using the SPSS software version 22 (SPSS Inc., Chicago, IL, USA). We checked the normal distribution of continuous variables using the Kolmogorov Smirnov test.

Data is reported as mean $\pm \mathrm{SD}$ for continuous or frequencies for categorical variables.

Pearson correlation coefficients between FoR score and other scores were calculated. Multivariate linear regression analysis by considering FoR score as dependent and age, sex, marital status, duration of the disease, and EDSS as independent variables was done.

A $p$ value less than 0.05 was considered significant.

\section{Results}

One hundred and sixty-five RRMS patients were enrolled. Female to male ratio was $(\mathrm{F} / \mathrm{M})=4.6$.

Mean age and disease duration were $35.3 \pm 8.6$ and $7.1 \pm 5$ years, respectively (Table 1).

Mean scores of social support, DASS, and FoR were 63.1 $\pm 16.8,16.4 \pm 13.4$, and $51.4 \pm 17.3$, respectively (Table 2). Depression, anxiety, and stress scores of DASS questionnaire were $6.1 \pm 5.8,5.1 \pm 4.3$, and $5.1 \pm 4.3$, respectively.

There was a significant negative correlation between social support and FoR score and also significant positive correlation between depression, anxiety, and stress components of DASS and FoR (Table 3).

Linear regression analysis by considering FoR as dependent variable and age, sex, marital status, duration of the disease, and EDSS as dependent variables showed that sex was a significant positive independent predictor (Table 4).

\section{Discussion}

To our knowledge, this is the first study evaluating fear of relapse and psychological well-being during the COVID-19 pandemic in patients with MS.

Nearly $85 \%$ of MS patients suffer from RR form of the MS disease [3]. Patients with RRMS suffer from unpredictable relapses which affect all aspects of their lives as new clinical signs appear or old signs worsen [15]. Relapses are associated with progression of the disease and damages to the multiple functions. Patients in the relapse phase of the disease suffer from impaired different aspects of quality of life [5]. Considering fear in patients with MS will help them to

Table 1 Basic characteristics of enrolled patients

\begin{tabular}{ll}
\hline Variables & Findings \\
\hline Age (year) & $35.3 \pm 8.6$ \\
Sex & \\
Female & $136(82.4 \%)$ \\
Male & $29(17.6 \%)$ \\
Marital status & \\
Single & $29(17.6 \%)$ \\
Married & $136(82.4 \%)$ \\
Education level & \\
$<12$ years & $78(47.2 \%)$ \\
$\geq 12$ years & $87(52.7 \%)$ \\
Occupation & \\
Employed & $103(62.4 \%)$ \\
Unemployed & $62(37.5 \%)$ \\
Disease duration (year) & $7.1 \pm 5$ \\
EDSS & $0.5 \pm 0.8$ \\
\hline
\end{tabular}


Table 2 Mean scores of questionnaires

\begin{tabular}{ll}
\hline Variables & Findings \\
\hline Social support & $63.1 \pm 16.8$ \\
DASS & $16.4 \pm 13.4$ \\
Depression & $6.1 \pm 5.8$ \\
Anxiety & $5.1 \pm 4.3$ \\
Stress & $8.8 \pm 5.5$ \\
FoR & $51.4 \pm 17.3$ \\
Feeling related to another relapse & $19.4 \pm 6.9$ \\
Physiological effects of relapse & $18.2 \pm 8.7$ \\
Fear associated with side effects of a relapse & $13.7 \pm 5.1$ \\
\hline
\end{tabular}

manage their affairs and improve their self-esteem which will result in better self-care and health improvement [16]. As relapses are unpredictable, they are source of stress and anxiety in the patients. Our results show that there were significant positive correlations between anxiety and stress scores with FoR score which confirms Khatibi et al.'s findings [10]. On the other hand, psychological well-being is needed to be stable and decrease relapse occurrence in MS patients.

The results also show that there was a weak significant negative correlation between social support and FoR score.

Patients with MS suffer from a range of psychological problems such as depression and anxiety which are reported in up to half of the patients $[17,18]$. It is obvious that stress could be the trigger of clinical relapses. Previous systematic review and meta-analysis showed that stress increases the risk of relapse by 4.8 (95\% CI: 1.69-14.1) [19].

Different items such as social support help MS cases to adjust to the disease easily and protect themselves against negative outcomes [20]. Increased social support will result in reduced depressive symptoms, lower anxiety experience, and better quality of life [21-23].

A recent study which was conducted in Iran showed that nearly one-third of MS patients were worried about COVIDrelated relapses during the pandemic stage [24] which may exacerbate their psychological disturbances. An Italian study showed that nearly $50 \%$ of MS patients suffered from moderate-to-severe anxiety during the pandemic stage [25]. Stojanov et al. reported that $72 \%$ of the MS patients were

Table 3 Correlation coefficients between scores and For score
Table 4 Linear regression analysis by considering FoR as dependent variable and other variables

\begin{tabular}{lll}
\hline Variable & $B$ & $p$ value \\
\hline Age & 0.06 & 0.7 \\
Sex & 7.3 & 0.04 \\
Marital status & -2.2 & 0.4 \\
Duration of the disease & 0.47 & 0.1 \\
EDSS & 0.8 & 0.6 \\
\hline
\end{tabular}

worried about getting to the hospital during the pandemic stage [26].

The results also show that sex is an independent demographic predictor of FoR which indicates that women experience more fear of relapse than men. One explanation for this finding could be sexual hormone change during life for a woman. For instance, meta-analysis showed that pregnancy decreases the risk of relapse $(\mathrm{RR}=0.56,95 \% \mathrm{CI}$ : $0.37-0.84)$ while postpartum period increases the risk $(1.43,95 \% \mathrm{CI}: 1.19$ to 1.72 ) and breast feeding period has no effect on relapse (RR, 1.32 [CI, 0.89 to 1.96]) [19]. It indicates that physicians should pay more attention to psychological well-being of female patients during this period.

Our study had some strengths. First, it is the first study assessing fear of relapse during the COVID-19 pandemic in patients with MS. Second, we assessed depression, anxiety, stress, and social support along with fear of relapse in enrolled patients.

\section{Conclusion}

Psychological well-being as well as fear of relapse should be considered in patients with MS during the COVID-19 pandemic stage.

\section{Declarations}

Conflict of interest The authors declare no competing interests.

Ethical approval None

Informed consent All completed informed consent forms.

\section{References}

1. Ghajarzadeh M et al (2014) Emotional intelligence (EI) of patients with multiple sclerosis (MS). Iran J Public Health 43(11):1550

2. AZIMI A et al (2019) Prevalence of sexual dysfunction in women with multiple sclerosis: a systematic review and meta-analysis. Maedica 14(4):408 
3. Weinshenker BG (1996) Epidemiology of multiple sclerosis. Neurol Clin 14(2):291-308

4. Halper J (2007) The psychosocial effect of multiple sclerosis: the impact of relapses. J Neurol Sci 256:S34-S38

5. Ghajarzadeh M, et al. (2016) Validity and reliability of the persian version of the perception de la scle'rose en plaques et de ses pousse'es questionnaire evaluating multiple sclerosis-related quality of life. International journal of preventive medicine 7.

6. Ozakbas S et al (2004) Correlations between multiple sclerosis functional composite, expanded disability status scale and healthrelated quality of life during and after treatment of relapses in patients with multiple sclerosis. J Neurol Sci 218(1-2):3-7

7. Santangelo $\mathrm{G}$ et al (2016) Anxiety in multiple sclerosis: psychometric properties of the State-Trait Anxiety Inventory. Acta Neurol Scand 134(6):458-466

8. Askari F et al (2014) Anxiety in patients with multiple sclerosis: association with disability, depression, disease type and sex. Acta Medica Iranica:889-892

9. Bonavita S, et al. (2020) Perceived stress and social support in a large population of people with multiple sclerosis recruited online through COVID 19 pandemic. European journal of neurology.

10. Khatibi A et al (2020) Development and validation of fear of relapse scale for relapsing-remitting multiple sclerosis: understanding stressors in patients. Frontiers in psychiatry 11:226

11. Costabile T, et al. (2020) COVID-19 pandemic and mental distress in multiple sclerosis: implications for clinical management. Eur $\mathrm{J}$ Neurol.

12. Bonavita $\mathrm{S}$ et al (2020) Digital triage for people with multiple sclerosis in the age of COVID-19 pandemic. Neurol Sci 41(5): 1007-1009

13. Asghari A, Saed F, Dibajnia P (2008) Psychometric properties of the Depression Anxiety Stress Scales-21 (DASS-21) in a nonclinical Iranian sample. Int J Psychol 2(2):82-102

14. Bagherian-Sararoudi R et al (2013) Psychometric properties of the Persian version of the multidimensional scale of perceived social support in Iran. Int J Prev Med 4(11):1277

15. McDonald WI et al (2001) Recommended diagnostic criteria for multiple sclerosis: guidelines from the International Panel on the diagnosis of multiple sclerosis. Annals of Neurology: Official
Journal of the American Neurological Association and the Child Neurology Society 50(1):121-127

16. Lorig KR et al (1999) Evidence suggesting that a chronic disease self-management program can improve health status while reducing hospitalization: a randomized trial. Med Care:5-14

17. Siegert R, Abernethy D (2005) Depression in multiple sclerosis: a review. J Neurol Neurosurg Psychiatry 76(4):469-475

18. Boeschoten RE et al (2017) Prevalence of depression and anxiety in multiple sclerosis: a systematic review and meta-analysis. J Neurol Sci 372:331-341

19. Xie $\mathrm{Y}$ et al (2020) Factors associated with relapses in relapsingremitting multiple sclerosis: a systematic review and meta-analysis. Medicine 99:27

20. Dennison L, Moss-Morris R, Chalder T (2009) A review of psychological correlates of adjustment in patients with multiple sclerosis. Clin Psychol Rev 29(2):141-153

21. Henry A et al (2019) Anxiety and depression in patients with multiple sclerosis: the mediating effects of perceived social support. Multiple Sclerosis and Related Disorders 27:46-51

22. Suh Y et al (2012) Physical activity, social support, and depression: possible independent and indirect associations in persons with multiple sclerosis. Psychol Health Med 17(2):196-206

23. Jaracz K et al (2010) Quality of life and social support in patients with multiple sclerosis. Neurol Neurochir Pol 44(4):358-365

24. Rezaeimanesh N, Sahraian MA, Moghadasi AN (2020) Evaluation of the Opinion of patients with multiple sclerosis on the outcomes of catching COVID-19 and its effects on the MS symptoms. Basic and clinical neuroscience 11(2):201

25. Zanghì A et al (2020) Mental health status of relapsing-remitting multiple sclerosis Italian patients returning to work soon after the easing of lockdown during COVID-19 pandemic: a monocentric experience. Mult Scler Relat Disord 46:102561

26. Stojanov A et al (2020) Psychological status of patients with relapsing-remitting multiple sclerosis during coronavirus disease2019 outbreak. Mult Scler Relat Disord 45:102407

Publisher's note Springer Nature remains neutral with regard to jurisdictional claims in published maps and institutional affiliations. 